\title{
Laparoscopic Roux en Y Gastric Bypass versus Sleeve Gastrectomy in the Management of Gastroesophageal Reflux Disease: A cohort study
}

\author{
Amr H Afifi, MD; Ibrahim Nabil Salama Elwardany, Mohamed Fayek Mahfouz \\ General Surgery Department, Faculty of Medicine, Ain Shams University, Egypt
}

\begin{abstract}
Background: Gastroesophageal reflux disease (GERD) is considered to be one of the most prevalent diseases of the gastrointestinal system all over the world. One of the major risk factors of GERD is morbid obesity. During the last decade, the prevalence of obesity has increased significantly around the world. Many studies have suggested the association between obesity and GERD symptoms. The effect of weight loss on decreasing the incidence and severity of GERD symptoms was found to be significant, and weight loss is considered to be one of the most important lines in managing GERD symptoms.
\end{abstract}

Aim: We aim to compare between the use of laparoscopic Sleeve Gastrectomy and Roux-en-Y gastric bypass in the Management of morbid obese patients with Gastroesophageal Reflux Disease GERD) and decide which approach is associated with the best short and long term effects on the prevalence and symptoms of Gastroesophageal reflux disease (GERD).

Patients and methods: A retrospective study is conducted in Ain Shams University Surgery Hospital during the period between March 2014 and July 2018. We included patients with Gastroesophageal reflux disease (GERD) to undergo bariatric surgery (RYGB vs Sleeve gastrectomy) in our hospital including a body mass index of more than $35 \mathrm{~kg} / \mathrm{m}_{2}$, aged between 18 and 65, with one or more co-morbidity and with failure of conservative treatment of obesity over a period of more than 2 years. We excluded patients with general contraindications to abdominal surgery, patients with grade 3 or 4 Gastroesophageal reflux disease (GERD), patients with Large size Hiatal hernias (more than $2 \mathrm{~cm}$ ), patients with history of previous major abdominal operations and patient with inflammatory bowel disease.

Results: A total number of 110 patients have been enrolled in our study. Both groups were similar in terms of age, sex, weight, average BMI and other co-morbidities such as diabetes, hypertension and dyslipidemia. The changes in GERD score were significantly higher in the LRYGB patients $(56.5 \%)$ compared to (41\%) in the LSG group. Patients, mostly in LSG group, experienced worsening of the GERD symptoms.

Conclusion: Both the most common bariatric surgery procedures were associated with improved GERD symptoms. We consider the Roux- en-Y gastric bypass to be the superior operation technique in improving GERD symptoms. Sleeve gastrectomy was associated with more de-novo GERD symptoms development. Some recent guidelines consider having a preoperative GERD symptom to be a contraindication to performing sleeve gastrectomy.

Key words: Comparative, Study, Laparoscopic, Roux En Y, Gastric Bypass, Sleeve Gastrectomy, Management, Gastroesophageal, Reflux, Disease.

\begin{abstract}
Introduction
Gastroesophageal reflux disease (GERD) is one of the most prevalent gastrointestinal diseases all over the world; Over $25 \%$ of adult population reports the typical symptoms of acid reflux and heartburn. ${ }^{1}$ GERD is a multifactorial disease where morbid obesity is one of its important risk factors. More than $50 \%$ of morbidly obese patients in the US present with one or more symptoms of GERD. ${ }^{2}$

In the last decade, the prevalence of obesity has increased significantly around the world. In the united states, the Center for Diseases Control and Prevention (CDC) reported that around $30 \%$ of the American adult population are considered morbidly obese, having a body mass index of more than 30 $\mathrm{kg} / \mathrm{m}_{2}$, this was associated with an exponential
\end{abstract}

increase in the obesity-related co-morbidities such as dyslipidemia, hypertension, type II diabetes mellitus, obstructive sleep apnea and many other obesity related problems. ${ }^{3}$

Multiple studies have suggested a two-sided relationship between obesity and GERD symptoms: obesity increases the risk of developing GERD symptoms, and GERD symptoms was more likely to be severe with increased obesity. Furthermore, the prevalence of GERD and GERD-like symptoms was reported to be $26 \%$ higher than normal populations. ${ }^{4}$ On the other hand, weight loss significantly decreased the incidence and severity of GERD symptoms, and it is considered to be one of the most important lines in managing GERD symptoms. ${ }^{5}$ 
Treatment of GERD includes a variety of options including adopting more healthy dietary patterns, lifestyle modifications, pharmacological treatments and anti-reflux procedures. ${ }^{6}$ The recent trends in the surgical literature highlighted the importance of bariatric surgery in managing and treating GERD as the only effective long-term management of GERD. ${ }^{7-9}$ Many studies compared the effects of various bariatric operations, especially Roux-en-Y gastric bypass (RYGB) and sleeve gastrectomy, on the incidence and symptom scales of GERD in morbidly obese patients. ${ }^{10-13}$

Sleeve gastrectomy was first introduced more than 15 years ago, with many surgeons preferring it for its simplicity, avoiding the need for the more complex intestinal anastomoses and bypasses, reducing the operative morbidity, complications and side effects. ${ }^{14}$ However, RYGB started to display more promising long-term results and was associated with more diabetes remission. ${ }^{15,16}$

Our study aims to compare the use of laparoscopic sleeve Gastrectomy and Roux-en-Y gastric bypass in the management of morbid obese patients with Gastroesophageal Reflux Disease and to decide which approach is yielding the best short and long-term outcome results on the prevalence and symptoms of GERD.

\section{Patients and methods}

We conducted a retrospective study, through reviewing medical records, in Ain Shams University Surgical Hospital, Cairo, Egypt during the period between March 2014 and July 2018. We included patients with a body mass index of more than 35 $\mathrm{kg} / \mathrm{m}_{2^{\prime}}$ aged between 18 and 65 , with one or more co-morbidity and and with failure of conservative treatment of obesity over a period of more than 2 years with GERD symptoms. We excluded patients with general contraindications to abdominal surgery, patients with grade 3 or 4 Gastroesophageal reflux disease (GERD), patients with Large size Hiatal hernias (more than $2 \mathrm{~cm}$ ), patients with history of previous major abdominal operations and patient with inflammatory bowel disease.

All the patients included in our study underwent a complete physical evaluation. Pre-operative preparation of the patients included history taking focusing on age, sex, weight, BMI, dietary habits, history of previous operations. All the patients underwent full general examination, full abdominal examination checking for scars of previous operations or abdominal wall hernias. General pre-operative laboratory investigations for all the patients included full blood count, prothrombin and thromboplastin time, liver function tests, albumin, liver AST, ALT, serum urea, serum creatinine, sodium and potassium, free T3, T4, TSH, Fasting blood sugar and HBA1C. Pre-operative investigations included ECG, CXR, upper GI endoscopy for detecting reflux esophagitis, Barrett's oesophagus and other upper GI tract abnormalities, 24-hour esophageal manometry and pelvi-abdominal Ultrasound for intra-abdominal and pelvic organ pathologies. Patients with cardiovascular troubles had ECHO. Patients with respiratory troubles as sleep apnea underwent respiratory function test and arterial blood gases.

Patients were telephoned to assess changes in GERD symptoms score post operatively.

The medical records of the patients were examined to record data regarding operation time, hospital stay duration, 90 days readmission rate, and postoperative complications: Including leakage, postoperative hemorrhage, infections, anastomotic stricture, marginal ulcers, and port site herniation.

\section{Operation techniques}

Standard operation techniques followed in Ain Shams university hospital were followed. For LSG, we used a 35-Fr bougie along the lesser curvature for calibration of the gastric tube; the longitudinal resection of the stomach was done from approximately 3 to $6 \mathrm{~cm}$ orally of the pylorus to the angle of His. The staple line was routinely over sewn with an absorbable running suture. LRYGB was performed with a 150-cm antecolic Roux-limb with either a linear stapled or circular stapled (25-mm) gastrojejunostomy according to the preference of the bariatric surgeon. A $50-\mathrm{cm}$ long biliopancreatic limb was chosen.

\section{Statistical Analysis}

Statistical analysis was performed using SPSS23.0. The continuous variables were presented as mean \pm SD, Descriptive statistics were used for demographic variables such as age, weight, and BMI. The student $t$ and Fisher exact 2-sided tests were used where appropriate. A comparison between the variables was done using the one-sample test. $P$-value is considered statistically significant if its value was less than 0.05 .

The assessment of GERD symptoms was measured according to a grading system where the absence of GERD symptoms was given a score of 0 , Intermittent or variable symptoms was associated with a score of 1 , Intermittent medication was given 2 , the use of $\mathrm{H} 2$ blockers or low dose PPI was associated with the score of 3 , the use of High dose PPI was given a score of 4 and patients who met the criteria for anti-reflux surgery, were given the score of 5 . 


\begin{tabular}{ll}
\hline Grade & Symptoms \\
\hline 0 & No symptoms of GERD \\
1 & Intermittent or variable symptoms, no medication \\
2 & Intermittent medication \\
3 & H2 blockers or low dose PPI \\
4 & High dose PPI \\
5 & Meet criteria for anti-reflux surgery, or prior surgery for GERD \\
\hline
\end{tabular}

\section{Results}

A total number of 110 patients, with a BMI of more than $35 \mathrm{~kg} / \mathrm{m} 2$ were enrolled in the study. The LSG group included50 patients and the LRYGB group included 60 patients. The follow-up rate after a period of 1 year was $94 \%$.Both groups were similar (non-significant) regarding the terms of age, sex, weight, average BMI and other co morbidities such as diabetes, hypertension and dyslipidemia were almost identical in both groups Table 1.

Table 1: Patient characteristics

\begin{tabular}{lccc}
\hline & LSG $(\mathbf{N}=\mathbf{5 0})$ & LRYGB (N=60) & P \\
\hline Age, mean \pm SD, Yr & $34.0 \pm 11.1$ & $42.1 \pm 11.2$ & NS* \\
Female, $\mathrm{n}(\%)$ & $36(72)$ & $43(71.6)$ & NS \\
Weight, mean \pm SD, Kg & $123.5 \pm 19.4$ & $124.8 \pm 19.8$ & NS \\
BMI, mean $\pm S D, k g / m 2$ & $43.6 \pm 5.3$ & $44.2 \pm 5.3$ & NS \\
Hypertension (\%) & 63 & 59 & NS \\
Diabetes (\%) & 24 & 26 & NS \\
Dyslipidemia (\%) & 67 & 51 & NS \\
GERD (\%) & 44 & 46 & NS \\
\hline
\end{tabular}

$*=$ non-significant.

The changes in GERD score were significantly higher in the LRYGB patients (56.5\%) than LSG group (41\%) ( $P$ <.05). Patients who experienced worsening of the GERD symptoms were mostly in the LSG group (22.7\%), while (3.5\%) in the LRYGB group. The rest of the sample showed no difference in their GERD status (Figure 1). The changes in the GERD score reveals that patients underwent Laparoscopic Roux-en-Y gastric bypass procedure were associated with better results compared to those underwent Laparoscopic sleeve gastrectomy $(\mathrm{P}<.0001)$.

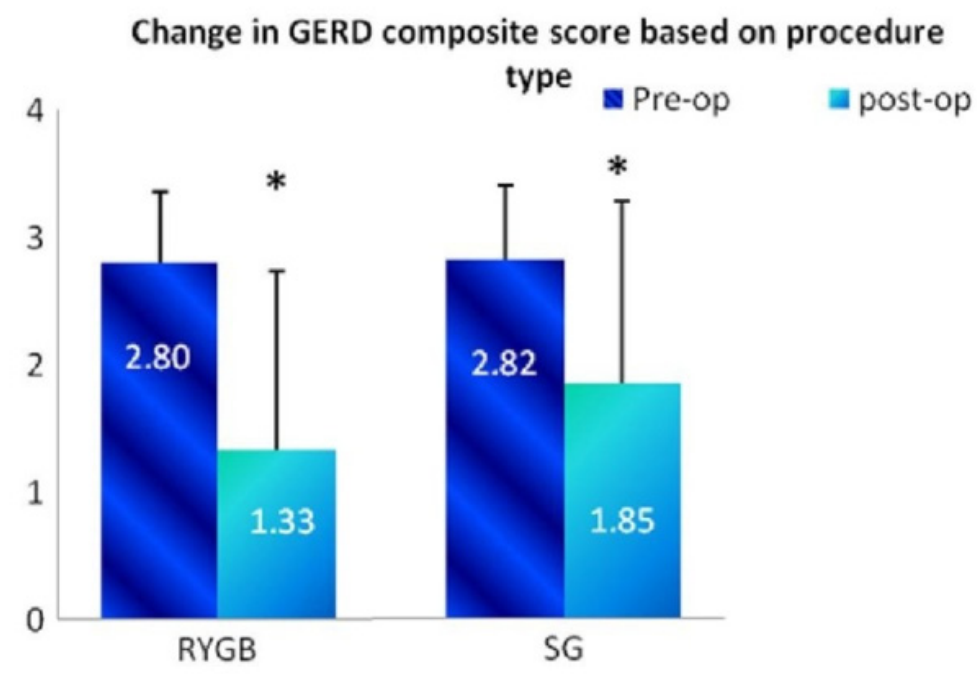

Fig 1: changes in GERD symptom score according to the type of the procedure. 
Regarding the change in GERD status, LRYGB had significantly improved GERD symptoms compared to LSG $(75 \%$ vs. $50 \%$, p-value 0.0008$)$, the rate of remission was higher in LRYGB (14\% vs 4\%, p-value 0.12 ), LSG was associated with more worsening of GERD symptoms ( $22 \%$ Vs 3.5\%). Table 2.

Table 2: The Change in GERD status according to each operation

\begin{tabular}{lccc}
\hline & LSG (N=50) & LRYGB (N=60) & P \\
\hline Preoperative GERD & 22 & 28 & 0.12 \\
Remission of GERD & 1 & 4 & 0.0008 \\
Improvement of GERD & 11 & 21 & \\
Worsening in GERD & 5 & 1 & \\
No change & 5 & 2 & \\
\hline
\end{tabular}

Postoperatively, and after follow-up, both groups were associated with improved co-morbidities. Regarding type 2 diabetes, $57.7 \%$ of patients in the
LSG group, and $67.9 \%$ of patients in the LRYGB were not taking any diabetes medication one year after the operation.

Table 3: Comparison of preoperative DM and remission of DM according to type of operation

\begin{tabular}{lcc}
\hline & LSG $(\mathbf{N}=\mathbf{5 0})$ & LRYGB $(\mathbf{N}=\mathbf{6 0})$ \\
\hline Preoperative Diabetes & 12 & 16 \\
Remission of DM & 7 & 11 \\
\hline
\end{tabular}

\section{Discussion}

In our study, patients undergoing both types of bariatric operations experienced improvement in their GERD symptoms. Our results showed a significant change in both GERD score and improvement of GERD symptoms in LRYGB group.

Howard et al. reported similar findings: An overall worsening of $82 \%$ of GERD symptoms in patients who underwent sleeve gastrectomy operations. ${ }^{17}$ Similarly, Tai et al. reported a significant increase in the symptoms of GERD after sleeve gastrectomy in 66 patents after one year of follow-up. ${ }^{18}$ On the other hand, Daes et al. reported a $48 \%$ decrease of GERD incidence of GERD symptoms after one year of performing sleeve gastrectomy. ${ }^{19}$ Soricelli et al. reported that GERD symptoms developed in about $22.9 \%$ of the patients undergoing sleeve gastrectomy alone, however when sleeve gastrectomy was combined with hiatal hernia repair, this risk dropped to $0 \%$ in his study. ${ }^{20}$

In 2011, Karmali et al. in a review of literature performed on 11 studies reported a decrease prevalence of GERD symptoms in a total of 7 studies, while the other 4 studies reported an increase in the incidence of GERD symptoms after performing a sleeve gastrectomy. ${ }^{12}$ Another study by Pallati et al. published in 2013 reported improvement in $41 \%$ of the patients who had previous GERD symptoms, worsening in $4.6 \%$ of the patients and newly developed GERD symptoms in $9.2 \%$ of the patients who had LSG. ${ }^{21}$

Murr et al showed that accurately performed LRYGB has resulted in a $94 \%$ improvement in typical GERD symptoms, with only $4 \%$ of the patients showing worsening symptoms. ${ }^{11}$ Accordingly, Schauer et al. reported similar significant results where GERD symptoms were decreased from $87 \%$ to $22 \%$ after performing LRYGB on morbidly obese patients with a BMI more than $35 \mathrm{~kg} / \mathrm{m}_{2}{ }^{22}$

The superiority of the Roux-en-Y gastric bypass operation can be explained by the nature of the operation, which includes creating a low-pressure gastric pouch connected to jejunum directly which results in reduced acid exposure and less acid and biliary reflux, leading to maximum and optimal results in reducing the incidence of GERD symptoms. ${ }^{23}$

Within a period of 6 months, patients in LRYGB group showed significant improvement in GERD symptoms postoperatively although no significant change in BMI is detected. A possible explanation of such results may be due to LRYGB creating a pouch and minimizing the exposure to acid and bile secretions. 
Our study has a few limitations, the retrospective nature of the study, and the relatively small sample may make our results hard to be generalized on a huge sample. The system used to measure the weight loss may be a little subjective and the GERD symptoms grading system can be a little confusing In cases of multiple drugs usage.

\section{Conclusion}

Both the most common bariatric surgery procedures were associated with improved GERD symptoms. We consider the Roux- en-Y gastric bypass to be the superior operation technique in improving GERD symptoms. Sleeve gastrectomy was associated with more de-novo GERD symptoms development. Some recent guidelines consider having a preoperative GERD symptom to be a contraindication to performing sleeve gastrectomy.

\section{References}

1. Tutuian R: Effects of bariatric surgery on gastroesophageal reflux. Curr Opin Gastroenterol.2014;30(4):434-438. doi:10.1097/ MOG.0000000000000083.

2. Prachand V, Alverdy JC: Gastroesophageal reflux disease and severe obesity: Fundoplication or bariatric surgery? World J Gastroenterol. 2010; 16: 3757-3761.

3. Peeters A, Barendregt JJ, Willekens $F$, et al: NEDCOM, the Netherlands Epidemiology and Demography Compression of Morbidity Research Group. Obesityin adulthood and its consequences for life expectancy: A life-table analysis. Ann Intern Med. 2003; 138: 24-23.

4. Ogden $\mathrm{CL}$, Yanovski SZ, Carroll MD, et al: Theepidemiology of obesity. Astroenterology. 2007; 132: 2087-2102.

5. El-Hadi M, Birch DW, Gill RS, et al: The effect of bariatric surgery on gastroesophageal reflux disease. Can J Surg. 2014; 57: 139-144.

6. De Groot NL, Burgerhart JS, Van De Meeberg PC, et al: Systematic review: The effects of conservative and surgical treatment for obesity on gastro-oesophageal reflux disease. Aliment Pharmacol Ther. 2009; 30: 1091-102.

7. Carlsson LM, Peltonen M, Ahlin S, et al: Bariatric surgery and prevention of type 2 diabetes in Swedishob.

8. Sjöström L, Narbro K, Sjöström CD, et al.

9. Sjöström $L$, Lindroos $A K$, Peltonen $M$, et al: Lifestyle, diabetes, and cardiovascular risk factors 10 years after bariatric surgery. $\mathbf{N}$ Engl $\boldsymbol{J}$ Med. 2004; 351: 268.
10. Klaus A, Gruber I, Wetscher G, et al: Prevalent esophageal body motility disorders underling aggravation of GERD symptoms in morbidly obese patients following adjustable gastric banding. Arch Surg. 2006; 141: 247-251.

11. Nelson LG, Gonzalez R, Haines K, et al: Amelioration of gastroesophageal reflux symptoms following Roux-en-Y gastric by pass for clinically significant obesity. Am Surg. 2005; 71: 950-953.

12. Chiu S, Birch DW, Shi X, et al: Effect of sleeve gastrectomy on gastroesophageal reflux disease: Asystematic review. Surg Obes Relat Dis. 2011; 7: 510-505.

13. De Jong JR, Besselink MGH, van Ramshorst $B$, et al: Effects of adjustable gastric banding on gastro esophageal reflux and esophageal motility: Asystematic review. Obesity Rev. 2010; 11: 297-305.

14. Iannelli $A$, Dainese $R$, Piche $T$, et al: Laparoscopic sleeve gastrectomy for morbid obesity. World J Gastroenterol. 2008; 14: 821827 [PMID:18240338].

15. Schauer PR, Bhatt DL, Kirwan JP, et al: Bariatric surgery versus intensive medical therapy for diabetes--3-year outcomes. N Engl J Med. 2014; 370: 2002-2013. [PMID:24679.

16. Li JF, Lai DD, Lin ZH, et al: Comparison of the long-term results of Roux-en-Y gastric bypass and sleeve gastrectomy for morbid obesity: A systematic review and metaanalysis of randomized and nonrandomized trials. Surg LaparoscEndosc.

17. Howard DD, Caban AM, Cendan JC, et al: Gastroesophageal reflux after sleeve gastrectomy in morbidly obese patients. Surg Obes Relat Dis. 2011; 7: 709-13.

18. Tai CM, Huang CK, Lee YC, et al: Increase in gastroesophageal reflux disease symptoms and erosiveesophagitis 1 year after laparoscopic sleeve rafter laparoscopic sleeve gastrectomy among obese adults. Surg Endosc. 2013;27:1260-6.

19. Daes J, Jimenez ME, Said N, et al: Laparoscopic sleeve gastrectomy: Symptoms of gastro esophageal reflux canbe reduced by changes in surgical technique. Obes Surg. 2012; 22: 18749.

20. Soricelli E, Iossa A, Casella G, et al: Sleeve gastrectomy and crural repairinobese patients with gastroesophageal reflux disease and/ orhiatalhernia. Surg Obes Relat Dis. Epub. 2012 Jun19. 
21. Pallati PK, Shaligram A, Shostrom VK, et al: Improvement in gastroesophageal reflux disease symptoms after various bariatric procedures: Review of the Bariatric Outcomes Longitudinal Database.Surge. 2014.

22. Frezza EE, Ikramuddin S, Gourash W, et al: Symptomaticimprovement ingastroesophageal reflux disease (GERD) following laparoscopic
Roux-en-Ygastric bypass. Surg Endosc. 2002; 16: 1027-31.

23. Prachand VN, Ward M, Alverdy JC: Duodenal switch provides superior resolution of metabolic comorbidities independent of weight loss in the super-obese (BMI Z50 kg/m ) compared with gastric bypass. J Gastrointest Surg. 2010; 14: 211- 20. 